\title{
Faux, falsification, pouvoir et société
}

Fakes, Falsification, Power and Society

\section{Emmanuel Poisson}

\section{CpenEdition}

\section{Journals}

Édition électronique

URL : http://journals.openedition.org/extremeorient/81

DOI : 10.4000/extremeorient.81

ISSN : 2108-7105

\section{Éditeur}

Presses universitaires de Vincennes

\section{Édition imprimée}

Date de publication : 1 octobre 2010

Pagination : 5-11

ISBN : 978-2-84292-263-4

ISSN : 0754-5010

\section{Référence électronique}

Emmanuel Poisson, «Faux, falsification, pouvoir et société », Extrême-Orient Extrême-Occident [En ligne], 32 | 2010, mis en ligne le 15 juin 2011, consulté le 21 septembre 2020. URL : http:// journals.openedition.org/extremeorient/81; DOI : https://doi.org/10.4000/extremeorient.81 


\title{
Faux, falsification, pouvoir et société
}

\author{
Emmanuel Poisson
}

La falsification - cantonnée pour l'essentiel à l'économie - minerait les fondements des sociétés contemporaines; elle aurait pour principal théâtre l'Extrême-Orient: de telles affirmations ont aujourd'hui valeur d'axiome dans l'opinion courante et les médias n'ont de cesse d'appeler à la vigilance ${ }^{1}$. La dimension marchande qui aujourd'hui domine l'approche du faux se traduit par un consensus dans sa formulation juridique. Fruit du travail d'historiens et d'anthropologues, le présent volume interroge la généalogie de cette conception très construite et s'inscrit dans un champ de recherches qui, ces dernières années, a connu un renouvellement de grande ampleur ${ }^{2}$. Il se propose de dessiner les contours d'un faux qui obéirait à d'autres déterminations que le marché. L'ensemble des textes, sans ambition exhaustive, donne à voir la pluralité des pratiques - le faux peut être réprimé, toléré ou revendiqué -, à scruter les mobiles des acteurs sociaux. Si elles se défient de toute réduction culturaliste, les contributions invitent pourtant à réfléchir sur le rapport des Chinois, Japonais et Vietnamiens à l'authenticité - une authenticité qui résiderait dans l'esprit et non

1. L'exposition «Contrefaçon, la vrai expo qui parle du faux» organisée à Paris par la Cité des sciences et de l'industrie du 20 avril 2010 au 13 février 2011 (http://www. cite-sciences.fr/francais/ala_cite/expositions/contrefacon/) traite des effets délétères de la falsification.

2. Voir en particulier: Umberto Eco, La Guerre du faux, Paris, Grasset, 1985. Nelson Goodman, «Art et authenticité» in Langages de l'art-une approche de la théorie des symboles, présenté et traduit de l'anglais par Jacques Morizot, Paris, Hachette, «Littératures», 2009, p. 135-161. Gérard Béaur, Hubert Bonin, Claire Lemercier (éd.), Fraude, contrefaçon et contrebande, de l'Antiquité à nos jours [colloque organisé à l'initiative de l'Association française des historiens économistes et du Comité pour l'histoire économique et financière de la France à Paris en novembre 2004], Genève, Droz, 2006. 
dans les choses ${ }^{3}$. Faux textes d'abdication, apocryphes religieux, généalogies truquées, faux artefacts archéologiques, fausses dates sur des monnaies officielles, hymens contrefaits. En dépit d'une telle hétérogénéité d'objets, les articles concourent à mieux cerner la signification du faux, les conditions de sa production et de sa circulation en Asie orientale. La réflexion procède d'un ensemble de paradoxes que l'on peut formuler ainsi. Le simulacre d'abdication dans la Chine $\mathrm{du}$ haut Moyen Âge ( $\mathrm{III}^{\mathrm{e}}-\mathrm{VI}^{\mathrm{e}} \mathrm{s}$.) semblait ne pas choquer les contemporains dans leur grande majorité (François Martin). Le pouvoir impérial en Chine a été conduit à apposer de fausses dates sur ses monnaies (François Thierry). Les auteurs étudient deux facettes de ce qui peut être qualifié de faux officiel. Guillaume Carré s'interroge sur la facilité avec laquelle les généalogies ont été truquées jusqu'au milieu du XVII ${ }^{\mathrm{e}}$ siècle au Japon. Loin de se limiter au milieu des parvenus en quête d'origine prestigieuse, le phénomène s'est généralisé à l'ensemble du corps social: individus, familles, villages, corps de métier, victimes d'ostracisme. Comment expliquer la coexistence entre nationalisme et universalisme au sein des nouvelles religions japonaises fondées sur des apocryphes? Tel est le paradoxe que soulève Jean-Pierre Berthon. Christian Lamouroux et Guillaume Carré se demandent comment dans la Chine des Song $\left(\mathrm{X}^{\mathrm{e}}-\mathrm{XIII}{ }^{\mathrm{e}} \mathrm{s}\right.$.) et le Japon d'Edo (XVII $-\mathrm{XIX}^{\mathrm{e}} \mathrm{s}$.) l'administration pouvait tirer profit de la circulation des faux (soies et soieries chinoises, remèdes japonais) malgré les prohibitions officielles. Pourquoi dans le Viêt Nam contemporain, le succès de l'hyménoplastie met-il en échec les préjugés les plus prégnants (Đinh Trọng Hiếu)? Comment interpréter l'inertie de l'Association japonaise d'archéologie face à une série de falsifications de grande ampleur d'outils paléolithiques courant sur près de trois décennies (Arnaud Nanta)?

Si l'on s'en tient à la terminologie dans la réglementation, alors le sens du faux ne souffre aucune équivoque: il est assimilé à une tromperie délibérée. Ainsi, sur un plan politique, dans la Chine médiévale, la confection d' «ordres forgés/contrefaits » était fréquente lors des coups de force. Ces textes ouvraient à leurs bénéficiaires les portes du palais impérial et légitimaient la destitution ou le meurtre d'un ministre rival. De tels ordres avaient pour office de duper ceux à qui ils étaient remis. L'intention maligne transparaît aussi dans les prescriptions du code des Tang relatives aux articles artisanaux. La falsification y prend deux visages - la «malfaçon» (ce qui n'est pas solide) et la «contrefaçon» (ce qui

3. Reprenant une intuition de Frederic W. Mote, Simon Leys a formulé cette hypothèse. Frederic W. Mote, “A Millenium of Chinese Urban History: Form, Time and Space Concepts in Soochow”, Rice University Studies, 59/4 (1973), p. 101-154. Simon Leys, «L'attitude des Chinois par rapport au passé», Commentaire, 10/39 (1987), p. 447-457, réédité in Essais sur la Chine, Paris, Robert Laffont, «Bouquins», 1998, p. 739-756. 
est inauthentique) - qui sont toutes deux délictueuses et appellent de ce fait une sanction par la loi. Le caractère licite d'un article, d'un objet, dépendait de leur conformité à des normes, d'où l'importance accordée aux mesures standardisées. Ainsi s'explique la vérification en Chine de la taille des étoffes ou la répression par la justice seigneuriale dans le Japon du XviII siècle des utilisateurs de mesures faussées. Enfin, les sources de la numismatique chinoise depuis le $\mathrm{V}^{\mathrm{e}}$ siècle, lorsqu'elles traitent du faux monnayage (d'époque), emploient des termes univoques - «faux» ou «mensonger» - quand la valeur intrinsèque (i.e. métallique) du multiple est trop éloignée de sa valeur faciale.

Toutefois, dans la pratique, le contrôle trahit vite ses limites. Celles-ci peuvent être d'ordre structurel ou tenir à la nature même de la surveillance. Dans la Chine des Tang ( $\mathrm{VII}^{\mathrm{e}}-\mathrm{X}^{\mathrm{e}} \mathrm{s}$.) et le Japon d'Edo, le contrôle était le plus souvent indirect. Le pouvoir, impérial dans le premier cas, seigneurial dans le second, le déléguait aux associations de producteurs et de marchands. L'impossibilité d'une surveillance par l'administration du shogun résultait du morcellement territorial de l'autorité politique hérité des guerres civiles de la fin du Moyen Âge. Sans doute la puissance publique dans la Chine et le Japon prémodernes s'arrogeait-elle le rôle de garant des étalons de mesure de poids, de taille ou de volume. Mais le contrôle ne pouvait être réduit à la vérification de la conformité à des standards. S'il était aisé de vérifier la taille d'une étoffe, il n'en allait pas de même de sa qualité, a fortiori de sa provenance. Comme le constatent Christian Lamouroux et Guillaume Carré, il n'est guère possible de parler d'un contrôle de qualité. L'analyse des compendiums de lois réalisés sur ordre des Tokugawa au XVIII ${ }^{e}$ siècle atteste que les vérifications sur l'«authenticité» des articles de consommation courante portaient souvent sur des gammes réduites. Certes, dans la seconde moitié du XVIII ${ }^{\mathrm{e}}$ siècle, les autorités shogunales imposèrent plusieurs dispositifs qui n'étaient pas sans rappeler nos systèmes d'appellation d'origine contrôlée pour certains produits. Mais l'enjeu était moins de vérifier leur qualité que de structurer des monopoles conçus pour servir leurs intérêts, en réservant la production et la commercialisation à certaines provinces. Les remarques qui précèdent ont conduit certains historiens chinois à interpréter la persistance des produits défectueux ou contrefaits comme l'expression emblématique de la faiblesse de l'organisation du contrôle des associations sur les produits. Tout aussi fluctuant apparait le contrôle de l'autorité publique sur les généalogies au Japon. À la fin du XII ${ }^{\mathrm{e}}$ siècle, la permission de prendre le nom d'un des quatre clans les plus prestigieux était l'une des marques de l'octroi du statut de vassal direct du shogun. Elle était alors accordée avec parcimonie. Mais aux

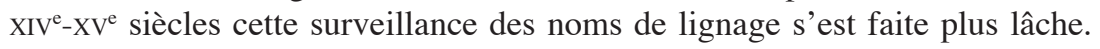
Nombre de samouraïs vendaient en ce temps leurs généalogies au profit de roturiers. De même la réussite économique a permis à de grands marchands de 
remettre en cause les hiérarchies des rangs et des origines en s'attribuant des noms de lignages renommés. Si la thèse du paléolithique japonais a longtemps pu triompher dans ce pays au $\mathrm{xx}^{\mathrm{e}}$ siècle, elle le doit en partie aux limites du contrôle du travail scientifique, limites procédant d'une double fragmentation, disciplinaire et structurelle. Arnaud Nanta estime qu'il est difficile de parler d'une archéologie au singulier. Les archéologues relèvent au Japon de structures différentes, qui peuvent être universitaires ou para-universitaires. Fondée en 1948, l'Association japonaise d'archéologie n'a longtemps eu pour ambition que de fédérer les chercheurs, laissant à chacune de ses composantes la validation ou l'invalidation des résultats scientifiques. Ce morcellement disciplinaire, qui n'est pas propre à ce pays, conjugue ses effets avec un caractère régional marqué de l'archéologie récente et, surtout, avec le poids des sociétés savantes locales, largement autonomes. Celles-ci contrôlent plus ou moins les publications destinées au grand public, notamment les manuels scolaires, par le biais des comités d'éducation locaux dont elles sont membres.

La falsification est-elle pour autant réductible à la tromperie? La majorité des articles montre qu'elle n'est pas nécessairement considérée comme répréhensible. Đinh Trọng Hiếu s'interroge sur cette « culture du faux » comme terrain favorable au succès contemporain de la falsification corporelle et de l'hymenoplastie en particulier. Nombreux sont les exemples de ce type au Viêt Nam. L'usage des «monnaies du Monde obscur»- đông à l'effigie du président Hô Chí Minh, dollars mais aussi euros -, faux billets destinés à être brûlés comme viatique pour les défunts, reste ainsi fort répandu. Le faux remplit également une fonction culinaire. L'imitation a alors pour double dessein de reproduire le goût et l'aspect du mets. Si le simulacre, le truquage ou la falsification stricto sensu visent tous à satisfaire une demande sociale, ils supposent une expertise au sens le plus large du terme et c'est l'un des intérêts du volume que de dégager les traits de ceux que l'on hésite à qualifier de faussaires, et ce pour deux raisons : ces hommes - généalogistes dans le Japon d'Edo, contrefacteurs d'actes d'abdication dans la Chine du haut Moyen Âge, intermédiaires dans les opérations commerciales et fiscales sous les Song - sont plutôt bien intégrés au tissu social; il n'y a pas alors dans ces deux pays de consensus sur la ligne de partage entre faux et authentique. Guillaume Carré observe que la généralisation du truquage des généalogies à l'ensemble de la société japonaise s'est accompagnée d'une diversification sociologique de l'expertise. Dans les luttes acharnées qu'ils se livraient, les seigneurs les plus puissants (daimyô) convoitaient les rangs de cour qui leur permettaient d'affirmer que leur pouvoir était légitimé par une autorité impériale ou shogounale, toute fictive qu'elle fût, mais censée toujours transcender les rivalités entre principautés guerrières. Cependant l'octroi de ces charges issues du régime des Codes impliquait une profondeur généalogique suffisamment 
forte pour rattacher les origines des daimyô au milieu curial. Ces derniers faisaient alors appel à des moines ou nobles de cour pour la rédaction d'arbres généalogiques conformes à leurs attentes. Dans la seconde moitié du XVII siècle, le milieu des généalogistes s'est ouvert à d'autres acteurs comme les savants ou les pseudo-érudits dont les rudiments de culture historique satisfaisaient une demande émanant alors de la notabilité villageoise: le travestissement généalogique visait à placer le bénéficiaire au niveau de l'aristocratie militaire. Dans la Chine des Six Dynasties, les deux rédacteurs de faux actes d'abdication que présente François Martin appartenaient à des clans puissants qui, apparentés à la famille impériale, avaient joué un rôle politique majeur tout au long des $\mathrm{IV}^{\mathrm{e}}$ et $v^{\mathrm{e}}$ siècles. Ils ont su conjuguer les atouts que leur procurait leur capital social à une vaste érudition et un sens politique aigu. Non moins négligeable était le rôle des intermédiaires dans la société Song. L'historiographie classique, qui les considère comme les principaux vecteurs de la corruption, est dépassée. Les malfaçons de la soie dont ils étaient les auteurs ne traduisent pas le délabrement moral de la société du temps mais peuvent être interprétées comme une parade à la pression fiscale.

L'ensemble des contributions met ainsi en lumière l'utilité sociale ou politique de la falsification. Pour les autorités chinoises du $\mathrm{X}^{\mathrm{e}}$ au XIII ${ }^{\mathrm{e}}$ siècle, tolérer dans une certaine mesure la production et la circulation de faux produits, c'était assurer la paix sociale par la régularité de l'approvisionnement urbain. Ainsi, sous les Song du Nord, Kaifeng hébergeait un marché de produits contrefaits ou défectueux. Le faux a pu être également pour le pouvoir impérial un outil de légitimation sur des territoires où il avait été contesté. François Thierry souligne à cet égard l'intérêt des monnaies «vraies» mais revêtues d'une fausse date, c'est-à-dire une date qui n'était pas celle de l'époque de leur émission ou une date en contradiction avec un autre élément de l'inscription monétaire. Les fausses datations ont pu être involontaires ou résulter d'un choix délibéré. C'est le dernier cas qui nous intéresse ici. Au XIX siècle, plusieurs régions du Xinjiang avaient, à la faveur de soulèvements, échappé à l'administration des Qing durant un laps de temps plus ou moins long. Aussi le monnayage de plusieurs ateliers pour certains règnes était-il insignifiant. Les autorités décidèrent d'uniformiser les quantités respectives de pièces en circulation par ateliers et par règnes afin de montrer que jamais le Xinjiang ne s'était soustrait à la souveraineté impériale: furent donc mises en circulation des «fausses » monnaies d'ères passées et/ou d'ateliers monétaires de villes devenues indépendantes. La falsification est ici revendiquée, pleinement assumée par le pouvoir. Il en va de même des textes analysés par François Martin. À la différence des «ordres contrefaits/forgés » dont l'intention frauduleuse était explicite, les actes d'abdication justifiaient le renversement du souverain. Nul n'ignorait à la cour que l'auteur du texte 
n'en était pas la victime. Sous les Six Dynasties, l'enjeu de ces textes - qui s'articulaient autour du discrédit de l'empereur déchu et de l'éloge du nouveau était de satisfaire les intérêts d'une coterie. Au Japon, du milieu du Xv siècle au début du XVII siècle, à l'époque des Provinces en guerre, le changement de nom n'était pas moins significatif: reprendre un patronyme célèbre mais tombé en déshérence était pour le représentant d'une maison guerrière l'expression d'une intention politique: s'approprier les territoires dont l'ancien lignage avait été le maître, et, en certains cas, parvenir au faîte de la hiérarchie militaire. Guillaume Carré souligne avec pertinence le parallélisme entre un tel geste et celui de certains fondateurs de dynasties en Chine, en Corée ou au Viêt Nam, qui reprirent le nom de royaumes disparus pour conforter leur légitimité politique. Dans le Japon du début du $\mathrm{xx}^{\mathrm{e}}$ siècle, la mobilisation par les nouvelles religions de textes apocryphes à caractère religieux a été un instrument de légitimation sociale de groupes marginalisés ou sur lesquels pesèrent des soupçons de déviances sectaires susceptibles de subvertir l'ordre public. Ainsi, certaines prophéties à caractère millénariste ont-elles été l'expression d'une quête de justice sociale dans le «Japon de l'arrière» où les plus déshérités, en particulier les femmes, avaient été les premières victimes de la modernisation du pays. De même, comme l'observe Arnaud Nanta, dans les années 1970-1990 les multiples affaires de falsification d'artefacts paléolithiques par des archéologues amateurs dans le département de Miyagi, amplement relayées par les médias locaux, ont été l'étendard d'une revendication identitaire d'un espace marginalisé. L'existence d'un paléolithique japonais a été un enjeu local mais aussi national. La réhabilitation en 1948 d'un premier ossement pourtant dénoncé en 1931 par certains archéologues japonais comme une falsification analogue à celle de Piltdown en Angleterre (1912-1953) s'inscrit dans une compétition chauvine entre les nations pour la découverte de l'homme fossile. Ces affaires dont la portée doit être relativisée - l'archéologie japonaise produit en grande majorité des travaux de qualité - ne sont toutefois pas dépourvues d'utilité : elles ont joué une fonction cathartique en contribuant à la mise en place de protocoles d'expertise plus exigeants. Đinh Trọng Hiêu s'est aussi penché sur l'utilité sociale de la falsification de l'hymen. Il voit dans l'hyménoplastie au xx siècle un signe de la rémanence d'un encadrement patriarcal et patrilinéaire de la société dont les règles ont été fixées au $\mathrm{XV}^{\mathrm{e}}$ siècle par le code des Lê ${ }^{4}$. Quand

4. Articles 388-400 in Quốc triêu hình luâtt [Lois pénales de la dynastie nationale], $\mathrm{xv}^{\mathrm{e}}$, Hà Nội, nhà xuất bản Chính trị Quốc gia, 1995, p. 144-148. Voir aussi Nguyên Ngoc Huy \& Ta Van Tài, The Lê Code: Law in Traditional Vietnam. A Comparative SinoVietnamese Legal Study with Historical-Juridical Analysis and Annotations. Athens, Ohio Univ. Press, 1987, vol. 1, p. 203-207 (traduction), vol. 2, p. 219-231 (analyse). 
une femme était convaincue d'adultère, la part de l'«encens et du feu»-i.e. les biens mobiliers ou immobiliers affectés au culte des ancêtres et à l'entretien de leur tombeau - risquait de passer à une branche cadette de la famille. La falsification répond ici à un dessein matrimonial: celui de jeunes filles qui veulent contracter un mariage honorable, alors qu'elles ont perdu leur virginité. L'auteur y décèle aussi une motivation d'ordre psychologique : la possibilité de «reconstruire» la personnalité féminine.

C'est à un nécessaire dépassement de la réification du faux et de l'authentique qu'invitent les auteurs. Au-delà de la diversité des champs considérés, les articles tendent à montrer que la césure entre le faux et l'authentique résulte d'un compromis instable entre les acteurs. L'enjeu de ce volume n'est pas tant l'étude de la répression du faux que la mise en lumière des rapports de force entre les groupes sociaux engagés. 